\title{
Berry-Esseen bounds for wavelet estimator in semiparametric regression model with linear process errors
}

\author{
Chengdong $\mathrm{Wei}^{1,2}$ and Yongming $\mathrm{Li}^{\mathrm{i}^{*}}$
}

\author{
* Correspondence: lym1019@163. \\ com \\ ${ }^{3}$ Department of Mathematics, \\ Shangrao Normal University, \\ Shangrao 334001, China \\ Full list of author information is \\ available at the end of the article
}

\begin{abstract}
Consider the semiparametric regression model $Y_{i}=x_{i} \beta+g\left(t_{i}\right)+\varepsilon_{i}, i=1, \ldots, n$, where the linear process errors $\varepsilon_{i}=\sum_{j=-\infty}^{\infty} a_{j} e_{i-j}$ with $\sum_{j=-\infty}^{\infty}\left|a_{j}\right|<\infty$, and $\left\{e_{i}\right\}$ are identically distributed and strong mixing innovations with zero mean. Under appropriate conditions, the Berry-Esseen type bounds of wavelet estimators for $\beta$ and $g(\cdot)$ are established. Our results obtained generalize the results of nonparametric regression model by Li et al. to semiparametric regression model.

Mathematical Subject Classification: 62G05; 62G08.
\end{abstract}

Keywords: wavelet estimator, semiparametric regression, strong mixing, Berry-Esseen bound

\section{Introduction}

Regression analysis is one of the most mature and widely applied branches of statistics. For a long time, however, its main theory has concerned parametric and nonparametric regressions. Recently, semiparametric regressions have received more and more attention. This is mainly because semiparametric regression reduces the high risk of misspecification relating to a fully parametric model and avoids some serious drawbacks of fully nonparametric methods.

In 1986, Engle et al. [1] first introduced the following semiparametric regression model:

$$
Y_{i}=x_{i} \beta+g\left(t_{i}\right)+\varepsilon_{i}, \quad i=1, \ldots, n,
$$

where $\beta$ is an unknown parameter of interest, $\left\{\left(x_{i}, t_{i}\right)\right\}$ are nonrandom design points, $\left\{y_{i}\right\}$ are the response variables, $g(\cdot)$ is an unknown function defined on the closed interval $[0,1]$, and $\left\{\varepsilon_{i}\right\}$ are random errors.

The model (1.1) has been extensively studied. When the errors $\left\{\varepsilon_{i}\right\}$ are independent and identically distributed (i.i.d.) random variables, Chen and Shiah [2], Donald and Dewey [3], and Hamilton and Truong [4] used various estimation methods to obtain estimators of the unknown quantities in (1.1) and discussed the asymptotic properties of these estimators. When $\left\{\varepsilon_{i}\right\}$ are MA $(\infty)$ errors with the form $\varepsilon_{i}=\sum_{j=0}^{\infty} a_{j} e_{i-j}$, where $\left\{e_{i}\right\}$ are i.i.d. random variables, $\left\{a_{j}\right\}$ satisfy $\sum_{j=-\infty}^{\infty}\left|a_{j}\right|<\infty$ and $\sup _{n} n \sum_{j=n}^{\infty}\left|a_{j}\right|<\infty$,

(c) 2012 Wei and Li; licensee Springer. This is an Open Access article distributed under the terms of the Creative Commons Attribution License (http://creativecommons.org/licenses/by/2.0), which permits unrestricted use, distribution, and reproduction in any medium, provided the original work is properly cited. 
the law of the iterated logarithm for the semiparametric least square estimator (SLSE) of $\beta$ and strong convergence rates of the nonparametric estimator of $g(\cdot)$ were discussed by Sun et al. [5]. The Berry-Esseen type bounds for estimators of $\beta$ and $g(\cdot)$ in model (1) under the linear process errors $\varepsilon_{i}=\sum_{j=-\infty}^{\infty} a_{j} e_{i-j}$ with identically distributed and negatively associated random variables $\left\{e_{i}\right\}$ were derived by Liang and Fan [6].

Let us now recall briefly the definition of strong-mixing dependence. A sequence $\left\{e_{i}\right.$, $i \in \mathbf{Z}$ \} is said to be strong mixing (or $\alpha$-mixing) if $\alpha(n) \rightarrow 0$ as $n \rightarrow \infty$, where $\alpha(n)=$ $\sup \left\{|\mathrm{P}(A B)-\mathrm{P}(A) \mathrm{P}(B)|: A \in \mathcal{F}_{-\infty}^{m} B \in \mathcal{F}_{m+n}^{\infty}\right\}$, and $\mathcal{F}_{n}^{m}$ denotes the $\sigma$-field generated by $\left\{e_{i}: m \leq i \leq n\right\}$.

For the properties of strong-mixing, one can read the book of Lin and Liu [7]. Recently, Yang and Li [8-10] and Xing et al. [11-13] established moment bounds and maximal moment inequality for partial sums for strong mixing sequences and their application. In this article, we study the Berry-Esseen type bounds for wavelet estimators of $\beta$ and $g(\cdot)$ in model (1.1) based linear process errors $\left\{\varepsilon_{i}\right\}$ satisfying the following basic assumption (A1). Our results obtained generalize the results in [14] to semiparametric regression model.

(A1) (i) Let $\varepsilon_{i}=\sum_{j=-\infty}^{\infty} a_{j} e_{i-j}$, where $\sum_{j=-\infty}^{\infty}\left|a_{j}\right|<\infty,\left\{e_{j}, j=0, \pm 1, \pm 2, \ldots\right\}$ are identically distributed and strong mixing random variables with zero mean.

(ii) For $\delta>0, \mathrm{E}\left|e_{0}\right|^{2+\delta}<\infty$ and mixing coefficients $\alpha(n)=O\left(n^{-\lambda}\right)$ for $\lambda>(2+\delta) / \delta$.

Now, we introduce wavelet estimators of $\beta$ and $g$ for model (1.1). Let $\beta$ be given, since $\mathrm{E} e_{i}=0$, we have $g\left(t_{i}\right)=E\left(y_{i}-x_{i} \beta\right), i=1, \ldots, n$. Hence a natural estimator of $g(\cdot)$ is

$$
g_{n}(t, \beta)=\sum_{j=1}^{n}\left(Y_{j}-x_{j} \beta\right) \int_{A_{j}} E_{m}(t, s) d s,
$$

where $A_{j}=\left[s_{j-1}, s_{j}\right]$ are intervals that partition $[0,1]$ with $t_{j} \in A_{j}$ and $0 \leq t_{1} \leq \cdots \leq t_{n}$ $\leq 1$, and wavelet kernel $E_{m}(t, s)$ can be defined by

$$
E_{m}(t, s)=2^{m} E_{0}\left(2^{m} t, 2^{m} s\right), E_{0}(t, s)=\sum_{j \in z} \varphi(t-j) \varphi(s-j)
$$

where $m=m(n)>0$ is a integer depending only on $n, \phi(\cdot)$ is father wavelet with compact support. Set

$$
\tilde{x}_{i}=x_{i}-\sum_{j=1}^{n} x_{j} \int_{A_{j}} E_{m}\left(t_{i}, s\right) d s, \tilde{y}_{i}=Y_{i}-\sum_{j=1}^{n} Y_{j} \int_{A_{j}} E_{m}\left(t_{i}, s\right) d s, S_{n}^{2}=\sum_{i=1}^{n} \tilde{x}_{i}^{2} .
$$

In order to estimate $\beta$, we seek to minimize

$$
S S(\beta)=\sum_{i=1}^{n}\left[Y_{i}-x_{i} \beta-g_{n}\left(t_{i}, \beta\right)\right]^{2}=\sum_{i=1}^{n}\left(\tilde{y}_{i}-\tilde{x}_{i} \beta\right)^{2} .
$$

The minimizer to (1.3) is found to be

$$
\hat{\beta}_{n}=S_{n}^{-2} \sum_{i=1}^{n} \tilde{x}_{i} \tilde{y}_{i}
$$


So, a plug-in estimator of the nonparametric component $g(\cdot)$, based on $\hat{\beta}_{n}$, is given by

$$
\hat{g}_{n}(t) \triangleq \hat{g}_{n}\left(t, \hat{\beta}_{n}\right)=\sum_{i=1}^{n}\left(Y_{i}-x_{i} \hat{\beta}_{n}\right) \int_{A_{i}} E_{m}(t, s) d s .
$$

In the following, the symbols $c, C, C_{1}, C_{2}, \ldots$ denote positive constants whose values may change from one place to another, $b_{n}=O\left(a_{n}\right)$ means $b_{n} \leq c a_{n},[x]$ denotes the integral part of $x,\left\|e_{i}\right\|_{r:}:=\left(E\left|e_{i}\right|^{r}\right)^{1 / r}, \Phi(u)$ represents the standard normal distribution function.

The article is organized as follows. In Section 2, we give some assumptions and main results. Sections 3 and 4 are devoted to the proofs of preliminary results. Proofs of theorems will be provided in Section 5. Some known results used in the proofs of preliminary and main results are appended in Appendix.

\section{Assumptions, notations and results}

At first we list some assumptions used in this article.

(A2) There exists a function $h(\cdot)$ defined on $[0,1]$ such that $x_{i}=h\left(t_{i}\right)+u_{i}$ and

(i) $\lim _{n \rightarrow \infty} n^{-1} \sum_{i=1}^{n} u_{i}^{2}=\sum_{0} \quad\left(0<\sum_{0}<\infty\right)$, (ii) $\max _{1 \leq i \leq n}\left|u_{i}\right|=O(1)$.

(iii) For any permutation $\left(j_{1}, \ldots, j_{n}\right)$ of the integers $(1, \ldots, n)$,

$$
\limsup _{n \rightarrow \infty} \frac{1}{\sqrt{n} \log n} \max _{1 \leq m \leq n}\left|\sum_{i=1}^{m} u_{j_{i}}\right|<\infty .
$$

(A3) The spectral density $f(\omega)$ of $\left\{\varepsilon_{i}\right\}$ satisfies $0<c_{1} \leq f(\omega) \leq c_{2}<\infty$, for $\omega \in(-\pi, \pi]$. (A4) Let $g(\cdot)$ and $h(\cdot)$ satisfy the Lipschitz condition of order 1 on $[0,1]$, and $h(\cdot) \in$ $H^{v}, v>\frac{3}{2}$, where $H^{v}$ is the Sobolev space of order $v$.

(A5) Scaling function $\phi(\cdot)$ is $\gamma$-regular ( $\gamma$ is a positive integer) and has a compact support, satisfies the Lipschitz condition of order 1 and $|\hat{\varphi}(\xi)-1|=O(\xi)$ as $\xi \rightarrow 0$, where $\hat{\varphi}$ denotes the Fourier transform of $\phi$.

(A6) $\max _{1 \leq i \leq n}\left|s_{i}-s_{i-1}\right|=O\left(n^{-1}\right)$.

(A7) There exists a positive constant $d_{1}$, such that $\min _{1 \leq i \leq n}\left(t_{i}-t_{i-1}\right) \geq d_{1} \cdot \frac{1}{n}$.

For the sake of convenience, we use the following notations. Let $p=p(n), q=q(n)$ denote positive integers such that $p+q \leq 3 n$ and $q p^{-1} \leq c<\infty$. Set

$$
\begin{gathered}
\sigma_{n 1}^{2}=\operatorname{Var}\left(\sum_{i=1}^{n} u_{i} \varepsilon_{i}\right), \sigma_{n 2}^{2}=\operatorname{Var}\left(\sum_{i=1}^{n} \varepsilon_{i} \int_{A_{i}} E_{m}(t, s) d s\right), u(n)=\sum_{j=n}^{\infty} \alpha^{\delta /(2+\delta)}(j) ; \\
\gamma_{1 n}=q p^{-1}, \quad \gamma_{2 n}=p n^{-1}, \quad \gamma_{3 n}=n\left(\sum_{|j|>n}\left|a_{j}\right|\right), \quad \gamma_{4 n}=n p^{-1} \alpha(q) ; \\
\lambda_{1 n}=q p^{-1} 2^{m}, \quad \lambda_{2 n}=p n^{-1} 2^{m}, \quad \lambda_{3 n}=\gamma_{3 n}, \lambda_{4 n}=\gamma_{4 n}, \quad \lambda_{5 n}=2^{-m / 2}+\sqrt{2^{m} / n} \log n ; \\
\mu_{n}(\rho, p)=\sum_{i=1}^{3} \gamma_{i n}^{1 / 3}+u(q)+\gamma_{2 n}^{\rho}+\gamma_{4 n}^{1 / 4} ; \\
v_{n}(m)=2^{-\frac{2 m}{3}}+\left(2^{m} / n\right)^{1 / 3} \log ^{2 / 3} n+2^{-m} \log n+n^{1 / 2} 2^{-2 m} .
\end{gathered}
$$


After these assumptions and notations we can formulate the main results as follows:

Theorem 2.1. Suppose that (A1)-(A7) hold. If $\rho$ satisfies

$$
0<\rho \leq 1 / 2, \quad \rho<\min \left\{\frac{\delta}{2}, \frac{\delta \lambda-(2+\delta)}{2 \lambda+(2+\delta)}\right\}
$$

then

$$
\sup _{u}\left|P\left(\frac{S_{n}^{2}\left(\hat{\beta}_{n}-\beta\right)}{\sigma_{n 1}} \leq u\right)-\Phi(u)\right| \leq C_{1}\left(\mu_{n}(\rho, p)+v_{n}(m)\right)
$$

Corollary 2.1 Under the same conditions as in Theorem 2.1 , if $\rho=1 / 3,2^{m}=O\left(n^{2 /}\right.$ $\left.{ }^{5}\right), \sup _{n \geq 1} n^{7 / 8}(\log n)^{-9 / 8} \sum_{|j|>n}\left|a_{j}\right|<\infty$ and $\delta>1 / 3, \lambda \geq \max \left\{\frac{2+\delta}{\delta}, \frac{7 \delta+14}{6 \delta-2}\right\}$, then for each $t \in[0,1]$, we have

$$
\left|P\left(\frac{S_{n}^{2}\left(\hat{\beta}_{n}-\beta\right)}{\sigma_{n 1}} \leq u\right)-\Phi(u)\right| \leq C_{2}\left(n^{-\frac{\lambda}{6 \lambda+7}}\right) .
$$

Theorem 2.2. Suppose that the conditions in Theorem 2.1 are satisfied. Let $n^{-1} 2^{m} \rightarrow$ 0 , then for each $t \in[0,1]$

$$
\sup _{u}\left|P\left(\frac{\hat{g}_{n}(t)-\mathrm{E} \hat{g}_{n}(t)}{\sigma_{n 2}} \leq u\right)-\Phi(u)\right| \leq C_{3}\left(\sum_{i=1}^{3} \lambda_{\text {in }}^{1 / 3}+u(q)+\lambda_{2 n}^{\rho}+\lambda_{4 n}^{1 / 4}+\lambda_{5 n}^{(2+\delta) /(3+\delta)}\right) .
$$

Corollary 2.2. Under the conditions of Theorem 2.2 with $\rho=1 / 3, \delta>2 / 3$, if $n^{-1} 2^{m}=$ $O\left(n^{-\theta}\right)$ with $\frac{\lambda+1}{2 \lambda+1}<\theta \leq 3 / 4$, and $\lambda>\frac{(2+\delta)(9 \theta-2)}{2 \theta(3 \delta-2)+2}$, then

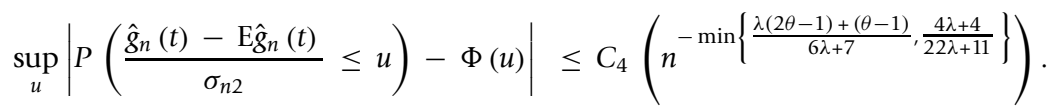

Remark 2.1. Let $\tilde{h}(t)=h(t)-\sum_{j=1}^{n} h\left(t_{j}\right) \int_{A_{j}} E_{m}(t, s) d s$, under the assumptions (A4)(A7) and by the relation (11) of the proof of Theorem 3.2 in [15], we obtain $\sup _{t}|\tilde{h}(t)|=O\left(n^{-1}+2^{-m}\right)$. Similarly, let $\tilde{g}(t)=g(t)-\sum_{j=1}^{n} g\left(t_{j}\right) \int_{A_{j}} E_{m}(t, s) d s$, then $\sup _{t}|\tilde{g}(t)|=O\left(n^{-1}+2^{-m}\right)$.

Remark 2.2. (i) By Corollary 2.1, the Berry-Esseen bound of the wavelet estimator $\hat{\beta}_{n}$ is near $O\left(n^{-\frac{1}{6}}\right)$ for sufficiently large $\lambda$, which is faster than the one in [16]) that can get $O\left(n^{-\delta / 4} \log n\right)$ for $\delta \leq 1 / 2$ or $O\left(n^{-1 / 8}\right)$ for $\delta>1 / 2$ for strong mixing sequence, but slower than the one in [6] for weighted estimate that can get $O\left(n^{-1 / 4}(\log n)^{3 / 4}\right)$.

(ii) From Corollary 2.2, the Berry-Esseen bound of the wavelet estimator $\hat{g}_{n}(\cdot)$ is near $O\left(\mathrm{n}^{-1 / 12}\right)$ for sufficiently large $\lambda$ and $\theta=3 / 4$.

\section{Some preliminary lemmas for $\hat{\boldsymbol{\beta}}_{n}$}

From the definition of $\hat{\beta}_{n}$ in (1.4), we write

$$
\begin{aligned}
S_{n \beta} & :=\sigma_{n 1}^{-1} S_{n}^{2}\left(\hat{\beta}_{n}-\beta\right)=\sigma_{n 1}^{-1}\left[\sum_{i=1}^{n} \tilde{x}_{i} \varepsilon_{i}-\sum_{i=1}^{n} \tilde{x}_{i} \sum_{j=1}^{n} \varepsilon_{j} \int_{A_{j}} E_{m}\left(t_{i}, s\right) d s+\sum_{i=1}^{n} \tilde{x}_{i} \tilde{g}_{i}\right] \\
& :=S_{n 1}+S_{n 2}+S_{n 3},
\end{aligned}
$$


where

$$
\begin{aligned}
& S_{n 1}= \sigma_{n 1}^{-1} \sum_{i=1}^{n} \tilde{x}_{i} \varepsilon_{i}=\sigma_{n 1}^{-1} \sum_{i=1}^{n} u_{i} \varepsilon_{i}+\sigma_{n 1}^{-1} \sum_{i=1}^{n} \tilde{h}_{i} \varepsilon_{i}-\sigma_{n 1}^{-1} \sum_{i=1}^{n} \varepsilon_{i}\left(\sum_{j=1}^{n} u_{j} \int_{A_{j}} E_{m}\left(t_{i}, s\right) d s\right) \\
&= S_{n 11}+S_{n 12}+S_{n 13}, \\
&\left|S_{n 2}\right|=\left|\sigma_{n 1}^{-1} \sum_{i=1}^{n} \tilde{x}_{i} \sum_{j=1}^{n} \varepsilon_{j} \int_{A_{j}} E_{m}\left(t_{i}, s\right) d s\right| \\
& \leq\left|\sigma_{n 1}^{-1} \sum_{i=1}^{n} u_{i}\left(\sum_{j=1}^{n} \varepsilon_{j} \int_{A j} E_{m}\left(t_{i}, s\right) d s\right)\right|+\left|\sigma_{n 1}^{-1} \sum_{i=1}^{n} \tilde{h}_{i}\left(\sum_{j=1}^{n} \varepsilon_{j} \int_{A_{j}} E_{m}\left(t_{i}, s\right) d s\right)\right| \\
& \quad+\left|\sigma_{n 1}^{-1} \sum_{i=1}^{n}\left(\sum_{l=1}^{n} u_{l} \int_{A_{l}} E_{m}\left(t_{i}, s\right) d s\right)\left(\sum_{j=1}^{n} \varepsilon_{j} \int_{A_{j}} E_{m}\left(t_{i}, s\right) d s\right)\right| \\
&=: S_{n 21}+S_{n 22}+S_{n 23}, \\
& S_{n 3}=\sigma_{n 1}^{-1} \sum_{i=1}^{n} \tilde{x}_{i} \tilde{g}_{i} .
\end{aligned}
$$

For $S_{n 11}$, we can write

$$
S_{n 11}=\sigma_{n 1}^{-1} \sum_{i=1}^{n} u_{i} \varepsilon_{i}=\sigma_{n 1}^{-1} \sum_{i=1}^{n} u_{i} \sum_{j=-n}^{n} a_{j} e_{i-j}+\sigma_{n 1}^{-1} \sum_{i=1}^{n} u_{i} \sum_{|j|>n} a_{j} e_{i-j}:=S_{n 111}+S_{n 112} .
$$

It is not difficult to see that

$$
S_{n 111}=\sum_{l=1-n}^{2 n} \sigma_{n 1}^{-1}\left(\sum_{i=\max \{1, l-n\}}^{\min \{n, l+n\}} u_{i} a_{i-l}\right) e_{l} \triangleq \sum_{l=1-n}^{2 n} Z_{n l} .
$$

Let $k=[3 n /(p+q)]$, then $S_{n 111}$ may be split as

$$
S_{n 111}=S_{n 111}^{\prime}+S_{n 111}^{\prime \prime}+S_{n 111}^{\prime \prime \prime}
$$

where

$$
\begin{aligned}
& S_{n 111}^{\prime}=\sum_{w=1}^{k} y_{1 n w}, \quad S_{n 111}^{\prime \prime}=\sum_{w=1}^{k} y_{1 n w^{\prime}}^{\prime} \quad S_{n 111}^{\prime \prime \prime}=y_{1 n k+1}^{\prime} \\
& y_{1 n w}=\sum_{i=k_{w}}^{k_{w}+p-1} Z_{n i}, \gamma_{1 n w}^{\prime}=\sum_{i=l_{w}}^{l_{w}+q-1} Z_{n i}, \gamma_{1 n k+1}^{\prime}=\sum_{i=k(p+q)-n+1}^{2 n} Z_{n i}, \\
& k_{w}=(w-1)(p+q)+1-n, l_{w}=(w-1)(p+q)+p+1-n, w=1, \ldots, k .
\end{aligned}
$$

From (3.1) to (3.6), we can write that

$$
S_{n \beta}=S_{n 111}^{\prime}+S_{n 111}^{\prime \prime}+S_{n 111}^{\prime \prime \prime}+S_{n 112}+S_{n 12}+S_{n 13}+S_{n 2}+S_{n 3} .
$$

Now, we establish the following lemmas with its proofs. 
Lemma 3.1. Suppose that (A1), (A2)(i), and (A3) hold, then

$$
c_{1} \pi n \leq \sigma_{n 1}^{2} \leq c_{2} \pi n, \quad c_{3} n^{-1} 2^{m} \leq \sigma_{n 2}^{2} \leq c_{4} n^{-1} 2^{m} .
$$

Proof. According to the proofs of (3.4) and Theorem 2.3 in [17], for any sequence $\{\gamma\}\}_{l \in N}$, we have

$$
2 c_{1} \pi \sum_{l=1}^{n} \gamma_{l}^{2} \leq E\left(\sum_{l=1}^{n} \gamma_{l} \varepsilon_{l}\right)^{2} \leq 2 c_{2} \pi \sum_{l=1}^{n} \gamma_{l}^{2},
$$

which implies the desired results by Lemma A.4 and assumption(A2)(i).

Lemma 3.2. Let assumptions (A1)-(A3), (A5), and (A6) be satisfied, then

$$
\begin{aligned}
& \mathrm{E}\left(S_{n 111}^{\prime \prime}\right)^{2} \leq C \gamma_{1 n}, \mathrm{E}\left(S_{n 111}^{\prime \prime \prime}\right)^{2} \leq C \gamma_{2 n}, \mathrm{E}\left(S_{n 112}\right)^{2} \leq C \gamma_{3 n} \\
& P\left(\left|S_{n 111}^{\prime \prime}\right| \geq \gamma_{1 n}^{1 / 3}\right) \leq C \gamma_{1 n}^{1 / 3}, P\left(\left|S_{n 111}^{\prime \prime \prime}\right| \geq \gamma_{2 n}^{1 / 3}\right) \leq C \gamma_{2 n}^{1 / 3}, P\left(\left|S_{n 112}\right| \geq \gamma_{3 n}^{1 / 3}\right) \leq C \gamma_{3 n}^{1 / 3} .
\end{aligned}
$$

Proof. By Lemmas 3.1 and A.1(i), and assumptions (A1)(i) and (A2)(i), we have

$$
\begin{aligned}
\mathrm{E}\left(S_{n 111}^{\prime \prime}\right)^{2} & \leq C \sum_{w=1}^{k} \sum_{i=l_{w}}^{l_{w}+q-1} \sigma_{n 1}^{-2}\left(\sum_{j=\max \{1, i-n\}}^{\min \{n, i+n\}} u_{i} a_{j-i}\right)^{2}\left\|e_{i}\right\|_{2+\delta}^{2} \\
& \leq C \sum_{w=1}^{k} \sum_{i=1_{w}}^{l_{w}+q-1} n^{-1}\left(\max _{1 \leq i \leq n}\left|u_{i}\right|\right)^{2}\left(\sum_{j=\max \{1, i-n\}}^{\min \{n, i+n\}}\left|a_{j-i}\right|\right)^{2} \\
& \leq C k q n^{-1}\left(\sum_{i=-\infty}^{\infty}\left|a_{j}\right|\right)^{2} \leq C q p^{-1}=C \gamma_{1 n}, \\
\mathrm{E}\left(S_{n 111}^{\prime \prime \prime}\right)^{2} & \leq C \sum_{i=k(p+q)-n+1}^{2 n} \sigma_{n 1}^{-2}\left(\sum_{j=\max \{1, i-n\}}^{\min \{n, i+n\}} u_{i} a_{j-i}\right)^{2}\left\|e_{i}\right\|_{2+\delta}^{2} \\
& \leq C \sum_{i=k(p+q)-n+1}^{2 n} n^{-1}\left(\max _{1 \leq i \leq n}\left|u_{i}\right|\right)^{2}\left(\sum_{j=\max \{1, i-n\}}^{\min \{n, i+n\}}\left|a_{j-i}\right|\right)^{2} \\
& \leq C[3 n-k(p+q)] n^{-1}\left(\sum_{j=-\infty}^{\infty}\left|a_{j}\right|\right)^{2} \leq C p n^{-1}=C \gamma_{2 n},
\end{aligned}
$$

and by the Cauchy-inequality

$$
\begin{aligned}
\mathrm{E}\left(S_{n 112}\right)^{2} & \leq C \sigma_{n 1}^{-2}\left(\sum_{i=1}^{n}\left|u_{i}\right|^{2}\right) E\left(\sum_{i=1}^{n}\left(\sum_{|j|>n} a_{j} e_{i-j}\right)^{2}\right) \leq C \sigma_{n 1}^{-2} n \sum_{i=1}^{n}\left(\sum_{|j|>n}\left|a_{j}\right|\right)^{2}\left\|e_{i}\right\|_{2+\delta}^{2} \\
& \leq C n\left(\sum_{|j|>n}\left|a_{j}\right|\right)^{2}=C \gamma_{3 n} .
\end{aligned}
$$

Then, from (3.9) to (3.11), the proof of (3.7) is complete, which implies the desired result (3.8) by the Markov-inequality. 
Lemma 3.3. Let assumptions (A1)-(A7) be satisfied, then

(a) $P\left\{\left|S_{n 12}\right| \geq C\left(n^{-1}+2^{-m}\right)^{2 / 3}\right\} \leq C\left(n^{-1}+2^{-m}\right)^{2 / 3}$.

(b) $P\left\{\left|S_{n 13}\right| \geq C\left(2^{m} n^{-1} \log ^{2} n\right)^{1 / 3}\right\} \leq C\left(2^{m} n^{-1} \log ^{2} n\right)^{1 / 3}$.

(c) $P\left\{\left|S_{n 21}\right| \geq C\left(2^{m} n^{-1} \log ^{2} n\right)^{1 / 3}\right\} \leq C\left(2^{m} n^{-1} \log ^{2} n\right)^{1 / 3}$.

(d) $P\left\{\left|S_{n 22}\right| \geq C\left(n^{-1}+2^{-m}\right)^{2 / 3}\right\} \leq C\left(n^{-1}+2^{-m}\right)^{2 / 3}$.

(e) $P\left\{\left|S_{n 23}\right| \geq C\left(2^{m} n^{-1} \log ^{2} n\right)^{1 / 3}\right\} \leq C\left(2^{m} n^{-1} \log ^{2} n\right)^{1 / 3}$.

(f) $S_{n 3} \leq C\left(2^{-m} \log n+n^{1 / 2} 2^{-2 m}\right)$.

Proof. (a) By assumption (A2), Remark 2.1 and Lemma 3.1, we get

$$
\mathrm{E}\left(S_{n 12}^{2}\right) \leq c_{2} \pi \sigma_{n 1}^{-2} \sum_{i=1}^{n}\left(\tilde{h}_{i}\right)^{2} \leq C\left(n^{-1}+2^{-m}\right)^{2},
$$

By this and the Markov inequality, we have

$$
P\left\{\left|S_{n 12}\right| \geq C\left(n^{-1}+2^{-m}\right)^{2 / 3}\right\} \leq C\left(n^{-1}+2^{-m}\right)^{2 / 3} .
$$

(b) Applying Lemmas 3.1, A.4, and A.5, we get that

$$
\begin{aligned}
\mathrm{ES}_{n 13}^{2} & \leq \sigma_{n 1}^{-2} \cdot c_{2} \sum_{i=1}^{n}\left(\sum_{j=1}^{n} u_{j} \int_{A_{j}} E_{m}\left(t_{i}, s\right) d s\right)^{2} \\
& \leq c_{2} \sigma_{n 1}^{-2} \cdot \max _{1 \leq i, j \leq n}\left|\int_{A_{j}} E_{m}\left(t_{i}, s\right) d s\right| \cdot \max _{1 \leq j \leq n} \sum_{i=1}^{n}\left|\int_{A_{j}} E_{m}\left(t_{i}, s\right) d s\right| \cdot\left(\max _{1 \leq m \leq n}\left|\sum_{i=1}^{m} u_{j_{i}}\right|\right)^{2} \\
& \leq C 2^{m} n^{-1} \log ^{2} n .
\end{aligned}
$$

Therefore

$$
P\left\{\left|S_{n 13}\right| \geq C\left(2^{m} n^{-1} \log ^{2} n\right)^{1 / 3}\right\} \leq C\left(2^{m} n^{-1} \log ^{2} n\right)^{1 / 3} .
$$

(c) Changing the order of summation in $\left\{S_{n 21}\right\}$, similarly to the calculation for $\mathrm{E} S_{n 13}^{2}$,

$$
\begin{aligned}
\mathrm{ES} S_{n 21}^{2} & \leq \sigma_{n 1}^{-2} \cdot c_{2} \sum_{j=1}^{n}\left(\sum_{i=1}^{n} u_{i} \int_{A_{j}} E_{m}\left(t_{i}, s\right) d s\right)^{2} \\
& \leq c_{2} \sigma_{n 1}^{-2} \cdot \max _{1 \leq i, j \leq n}\left|\int_{A_{j}} E_{m}\left(t_{i}, s\right) d s\right| \cdot \max _{1 \leq i \leq n} \sum_{j=1}^{n}\left|\int_{A_{j}} E_{m}\left(t_{i}, s\right) d s\right| \cdot\left(\max _{1 \leq m \leq n}\left|\sum_{i=1}^{m} u_{j_{i}}\right|\right)^{2} \\
& \leq C 2^{m} n^{-1} \log ^{2} n .
\end{aligned}
$$

Therefore, we obtain that

$$
P\left\{\left|S_{n 21}\right| \geq C\left(2^{m} n^{-1} \log ^{2} n\right)^{1 / 3}\right\} \leq C\left(2^{m} n^{-1} \log ^{2} n\right)^{1 / 3} .
$$


(d) Similarly, by Lemmas 3.1, A.4, A.5, and Remark 2.1, we get that

$$
\begin{aligned}
\mathrm{ES}_{n 22}^{2} & \leq c_{2} \sigma_{n 1}^{-2} \cdot \sum_{i=1}^{n}\left(\sum_{j=1}^{n} \tilde{h}_{j} \int_{A_{i}} E_{m}\left(t_{j}, s\right) d s\right)^{2} \\
& \leq c_{2} \sigma_{n 1}^{-2} \cdot\left(\sup _{t_{j}}\left|\tilde{h}_{j}\right|\right)^{2} \sum_{i=1}^{n}\left(\sum_{j=1}^{n}\left|\int_{A_{i}} E_{m}\left(t_{j}, s\right) d s\right|\right)\left(\sum_{j=1}^{n}\left|\int_{A_{i}} E_{m}\left(t_{i}, s\right) d s\right|\right) \\
& \leq c_{2} \sigma_{n 1}^{-2} \cdot\left(\sup _{t_{j}}\left|\tilde{h}_{j}\right|\right)^{2} \cdot n \leq C\left(n^{-1}+2^{-m}\right)^{2} .
\end{aligned}
$$

Thus, we have

$$
P\left\{\left|S_{n 22}\right| \geq C\left(n^{-1}+2^{-m}\right)^{2 / 3}\right\} \leq C\left(n^{-1}+2^{-m}\right)^{2 / 3} .
$$

(e) We write that

$$
S_{n 23}=\sigma_{n 1}^{-1} \sum_{j=1}^{n}\left\{\sum_{i=1}^{n} \int_{A_{j}} E_{m}\left(t_{i}, s\right) d s\left(\sum_{l=1}^{n} \int_{A_{l}} E_{m}\left(t_{i}, s\right) u_{l} d s\right)\right\} \varepsilon_{j} .
$$

Similarly to the calculation for $\mathrm{ES}_{n 13}^{2}$ by (3.13), Lemmas 3.1, A.4, and A.5, we obtain that

$$
\begin{aligned}
\mathrm{ES}_{n 23}^{2} \leq c_{2} \pi \sigma_{n 1}^{-2} \sum_{j=1}^{n}\left[\sum_{i=1}^{n} \int_{A_{j}} E_{m}\left(t_{i}, s\right) d s\left(\sum_{l=1}^{n} \int_{A_{l}} E_{m}\left(t_{i}, s\right) u_{l} d s\right)\right]^{2} \\
\leq c_{2} \pi \sigma_{n 1}^{-2} \max _{1 \leq i, j \leq n} \int_{A_{i}} E_{m}\left(t_{j}, s\right) d s \cdot \max _{1 \leq j \leq n} \sum_{i=1}^{n} \int_{A_{i}} E_{m}\left(t_{j}, s\right) d s \\
\cdot\left(\max _{1 \leq l \leq n} \sum_{j=1}^{n} \int_{A_{l}} E_{m}\left(t_{j}, s\right) d s \cdot \max _{1 \leq m \leq n}\left|\sum_{i=1}^{m} u_{j_{i}}\right|\right)^{2} \leq C \cdot 2^{m} n^{-1} \log ^{2} n .
\end{aligned}
$$

Hence, we have

$$
P\left\{\left|S_{n 23}\right| \geq C\left(2^{m} n^{-1} \log ^{2} n\right)^{1 / 3}\right\} \leq C\left(2^{m} n^{-1} \log ^{2} n\right)^{1 / 3} .
$$

(f) By assumption (A2), Remarks 2.1, Lemma A.5, and the Abel inequality, we have

$$
\begin{aligned}
\sigma_{n 1} S_{n 3} & \leq\left|\sum_{i=1}^{n} u_{i} \tilde{g}_{i}\right|+\left|\sum_{i=1}^{n} \tilde{h}_{i} \tilde{g}_{i}\right|+\left|\sum_{i=1}^{n}\left(\left|\sum_{j=1}^{n} u_{j}\right| \int_{A_{j}} E_{m}\left(t_{i}, s\right) d s\right) \tilde{g}_{i}\right| \\
& \leq c\left\{\max _{1 \leq i \leq n}\left|\tilde{g}_{i}\right| \max _{1 \leq k \leq n}\left|\sum_{i=1}^{k} u_{j i}\right|+n \max _{1 \leq i \leq n}\left|\tilde{h}_{i}\right| \max _{1 \leq i \leq n}\left|\tilde{g}_{i}\right|\right. \\
& \left.+\max _{1 \leq i \leq n}\left|\tilde{g}_{i}\right| \max _{1 \leq j \leq n} \sum_{i=1}^{n} \int_{A_{j}}\left|E_{m}\left(t_{i}, s\right)\right| d s \max _{1 \leq k \leq n} \sum_{i=1}^{k}\left|u_{j_{i}}\right|\right\} \\
& =C_{1}\left(n^{-1}+2^{-m}\right) \sqrt{n} \log n+C_{2} n\left(n^{-1}+2^{-m}\right)^{2} .
\end{aligned}
$$


Thus, by Lemma 3.1 we obtain

$$
S_{n 3} \leq C_{1}\left(n^{-1}+2^{-m}\right) \log n+C_{2} \sqrt{n}\left(n^{-1}+2^{-m}\right)^{2} \leq C\left(2^{-m} \log n+n^{1 / 2} 2^{-2 m}\right) .
$$

Therefore, the desired result (3.12) follows from (3.13)-(3.18) immediately.

Lemma 3.4. Suppose that (A1)-(A3), (A5), and (A6) hold. Set $s_{n}^{2} \triangleq \sum_{w=1}^{k} \operatorname{Var}\left(y_{1 n w}\right)$, then

$$
\left|s_{n}^{2}-1\right| \leq C\left(\gamma_{1 n}^{1 / 2}+\gamma_{2 n}^{1 / 2}+\gamma_{3 n}^{1 / 2}+u(q)\right) \text {. }
$$

Proof. Let $\Gamma_{n}=\sum_{1 \leq i<j \leq k} \operatorname{Cov}\left(y_{1 n i}, y_{1 n j}\right)$, then $s_{n}^{2}=\mathrm{E}\left(S_{n 111}^{\prime}\right)^{2}-2 \Gamma_{n}$. By (3.5) and (3.6), it is easy to verify that $\mathrm{E}\left(S_{n 11}\right)^{2}=1$, and

$$
\mathrm{E}\left(S_{n 111}^{\prime}\right)^{2}=1+\mathrm{E}\left(S_{n 111}^{\prime \prime}+S_{n 111}^{\prime \prime \prime}+S_{n 112}\right)^{2}-2 \mathrm{E}\left[S_{n 11}\left(S_{n 111}^{\prime \prime}+S_{n 111}^{\prime \prime \prime}+S_{n 112}\right)\right] .
$$

According to Lemma 3.2, the $C_{r}$-inequality and the Cauchy-Schwarz inequality

$$
\mathrm{E}\left(S_{n 111}^{\prime \prime}+S_{n 111}^{\prime \prime \prime}+S_{n 112}\right)^{2} \leq C\left(\gamma_{1 n}+\gamma_{2 n}+\gamma_{3 n}\right),
$$

and

$$
\left|\mathrm{E}\left[S_{n 11}\left(S_{n 111}^{\prime \prime}+S_{n 111}^{\prime \prime \prime}+S_{n 112}\right)\right]\right| \leq C\left(\gamma_{1 n}^{1 / 2}+\gamma_{2 n}^{1 / 2}+\gamma_{3 n}^{1 / 2}\right) .
$$

Thus, we obtain

$$
\left|\mathrm{E}\left(S_{n 111}^{\prime}\right)^{2}-1\right| \leq C\left(\gamma_{1 n}^{1 / 2}+\gamma_{2 n}^{1 / 2}+\gamma_{3 n}^{1 / 2}\right) .
$$

On the other hand, from Lemma 1.2.4 in [7], Lemmas 3.1 and A.4(iv), we can estimate

$$
\begin{aligned}
& \left|\Gamma_{n}\right| \leq \sum_{1 \leq i \leq j \leq k} \sum_{s_{1}=k_{i}}^{k_{i}+p-1} \sum_{t_{1}=k_{j}}^{k_{j}+p-1}\left|\operatorname{Cov}\left(Z_{n s_{1}}, Z_{n t_{1}}\right)\right| \\
& \leq \frac{C}{n} \sum_{1 \leq i \leq \leq \leq k} \sum_{s_{1}=k_{i}}^{k_{i}+p-1} \sum_{t_{1}=k_{j}} \sum_{u=\max \left(1, s_{1}-n\right\}}^{\min \left\{n, n s_{1}+n\right\}} \sum_{v=\max \left\{1, t_{1}-n\right\}}^{\min \left\{n, t_{1}+n\right\}}\left|u_{u-s_{1}} u_{v-t_{1}}\right|\left|a_{u-s_{1}} a_{v-t_{1}}\right|\left|\operatorname{Cov}\left(e_{s_{1}}, e_{t_{1}}\right)\right| \\
& \leq \frac{C}{n} \sum_{1 \leq i \leq j \leq k} \sum_{s_{1}=k_{i}}^{k_{i}+p-1} \sum_{t_{1}=k_{j}} \sum_{u=\max \left(1, s_{1}-n\right\}}^{\min \left\{n, s_{1}+n\right\}} \sum_{v=\max \left\{1, t_{1}-n\right\}}^{\min \left\{n t_{1}+n\right\}}\left|a_{u-s_{1}} a_{v-t_{1}}\right| \alpha^{\delta /(2+\delta)}\left(t_{1}-s_{1}\right)\left\|e_{t_{1}}\right\|_{2+\delta} \cdot\left\|e_{s_{1}}\right\|_{2+\delta} \\
& \leq \frac{C}{n} \sum_{i=1}^{k-1} \sum_{s_{1}=k_{i}}^{k_{i}+p-1} \sum_{\left.u=\max \left\{1, s_{1}-n\right\}\right\}}^{\min \left\{\left\{, s_{1}+n\right\}\right.} \sum_{j=i+1}^{k} \sum_{t_{1}=k_{j}}^{k_{j}+p-1} \sum_{v=\max \left\{1, t_{1}-n\right\}}^{\min \left\{n, t_{1}+n\right\}} \alpha^{\delta /(2+\delta)}\left(t_{1}-s_{1}\right)\left|a_{u-s 1} a_{v-t_{1}}\right| \\
& \leq \frac{C}{n} \sum_{i=1}^{k-1} \sum_{s_{1}=k_{i}}^{k_{i}+p-1} \sum_{j=i+1}^{k} \sum_{t_{1}=k_{j}}^{k_{j}+p-1} \alpha^{\delta /(2+\delta)}\left(t_{1}-s_{1}\right) \\
& \leq \frac{C}{n} \sum_{i=1}^{k-1} \sum_{s_{1}=k_{i}}^{k_{i}+p-1} \sum_{t_{1}:\left|t_{1}-s_{1}\right| \geq q} \alpha^{\delta /(2+\delta)}\left(t_{1}-s_{1}\right) \leq C k p n^{-1} u(q) \leq C u(q) .
\end{aligned}
$$

Therefore, by (3.19) and (3.20), it follows that

$$
\left|s_{n}^{2}-1\right| \leq\left|E\left(S_{1 n}^{\prime}\right)^{2}-1\right|+2\left|\Gamma_{n}\right| \leq C\left(\gamma_{1 n}^{1 / 2}+\gamma_{2 n}^{1 / 2}+\gamma_{3 n}^{1 / 2}+u(q)\right) \text {. }
$$


Assume that $\left\{\eta_{1 n w}: w=1, \ldots, k\right\}$ are independent random variables, and its distribution is the same as that of $\left\{y_{1 n w}, w=1, \ldots, k\right\}$. Set $T_{n}=\sum_{w=1}^{k} \eta_{1 n w}$, $B_{n 1}^{2}=\sum_{w=1}^{k} \operatorname{Var}\left(\eta_{1 n w}\right)$. Clearly $B_{n 1}^{2}=s_{n 1}^{2}$. Then, we have the following lemmas:

Lemma 3.5. Let assumptions (A1)-(A3), (A5), (A6), and (2.1) hold, the

$$
\sup _{u}\left|P\left(T_{n} / B_{n 1} \leq u\right)-\Phi(u)\right| \leq C \gamma_{2 n}^{\rho} .
$$

Proof. By the Berry-Esseen inequality (see [18], Theorem 5.7]), we have

$$
\sup _{u}\left|P\left(T_{n} / B_{n 1} \leq u\right)-\Phi(u)\right| \leq C \frac{\sum_{w=1}^{k} E\left|y_{1 n w}\right|^{r}}{B_{n 1}^{r}}, \quad \text { for } 2<r \leq 3 .
$$

From $(2.1)$, we have $0<2 \rho \leq 1,0<2 \rho<\delta$, and, $(2+\delta) / \delta<(1+\rho)(2+\delta) /(\delta-2 \rho)$ $<\lambda$. Let $r=2(1+\rho), \tau=\delta$-2 $\rho$, then $r+\tau=2+\delta$, and $\frac{r(r+\tau)}{2 \tau}=\frac{(1+\rho)(2+\delta)}{\delta-2 \rho}<\lambda$. According to Lemmas 3.1 and A.1(ii), and the $C_{r}$-inequality, taking $\varepsilon=\rho$, we get that

$$
\begin{aligned}
\sum_{w=1}^{k} \mathrm{E}-\left.\gamma_{1 n w}\right|^{r} \leq & \left.C \sum_{w=1}^{k} p^{\rho} \sum_{j=k_{w}}^{k_{w}+p-1} \sum_{i=\max \{1, j-n\}}^{\min \{n, j+n\}} \sigma_{n 1}^{-1} u_{i} a_{i-j}\right|^{r} \mathrm{E}\left|e_{j}\right|^{r} \\
& +\left[\sum_{j=k_{w}}^{k_{w}+p-1}\left(\sum_{i=\max \{1, j-n\}}^{\min \{n, j+n\}} \sigma_{n 1}^{-1} u_{i} a_{i-j}\right)^{2}\left\|e_{i}\right\|_{2+\delta}^{2}\right]^{r / 2} \\
\leq & C \sigma_{n 1}^{-r} k p^{1+\rho} \leq C \gamma_{2 n}^{\rho} .
\end{aligned}
$$

Therefore, from Lemma 3.4, relations (3.21) and (3.22), we obtain the result.

Lemma 3.6. Suppose that the conditions in Lemma 3.5 are satisfied, then

$$
\sup _{u}\left|P\left(S_{n 111}^{\prime} \leq u\right)-P\left(T_{n} \leq u\right)\right| \leq C\left\{\gamma_{2 n}^{\rho}+\gamma_{4 n}^{1 / 4}\right\} .
$$

Proof. Let $\varphi_{1}(t)$ and $\psi_{1}(t)$ be the characteristic functions of $S_{n 111}^{\prime}$ and $T_{n 1}$, respectively.

Since

$$
\psi_{1}(t)=\mathrm{E}\left(\exp \left\{\mathbf{i} t T_{n 1}\right\}\right)=\prod_{w=1}^{k} \mathrm{E} \exp \left\{\mathbf{i} t \eta_{1 n w}\right\}=\prod_{w=1}^{k} \mathrm{E} \exp \left\{\mathbf{i} t y_{1 n w}\right\},
$$

then from Lemmas A.1(i), A.2, and 3.1, it follows that

$$
\begin{aligned}
\left|\phi_{1}(t)-\psi_{1}(t)\right| & \leq C|t| \alpha^{1 / 2}(q) \sum_{w=1}^{k}\left\|\gamma_{1 n w}\right\|_{2} \\
& \leq C|t| \alpha^{1 / 2}(q) \sum_{w=1}^{k}\left\{E\left[\sum_{i=k_{w}}^{k_{w}+p-1} \sigma_{n}^{-1}\left(\sum_{j=\max (1, i-n\}}^{\min \{n, i+n\}} u_{i} a_{j-i}\right) e_{i}\right]^{2}\right\}^{1 / 2} \\
& \leq C|t| \alpha^{1 / 2}(q) \sum_{w=1}^{k}\left[\sum_{i=k_{w}}^{k_{w}+p-1} \sigma_{n}^{-2}\left(\sum_{j=\max \{1, i-n\}}^{\min \{n, i+n\}}\left|u_{i} a_{j-i}\right|\right)^{2}\left(E\left|e_{i}\right|^{2+\delta}\right)^{2 / 2+\delta}\right]^{1 / 2} \\
& \leq C|t| \alpha^{1 / 2}(q)\left[k \sum_{w=1}^{k} \sum_{i=k_{w}}^{k_{w}+p-1} \sigma_{n}^{-2}\right]^{1 / 2} \leq C|t| \alpha^{1 / 2}(q) k p^{1 / 2} n^{-1 / 2} \\
& \leq C|t|(k \alpha(q))^{1 / 2}=C|t| \gamma_{4 n}^{1 / 2} .
\end{aligned}
$$


Therefore

$$
\int_{-T}^{T}\left|\frac{\phi_{1}(t)-\psi_{1}(t)}{t}\right| d t \leq C \gamma_{4 n}^{1 / 2} T
$$

As in the calculation of (4.7) in [14], using Lemma 3.5, we have

$$
T \sup _{u} \int_{|\gamma| \leq c / T}\left|P\left(T_{n} \leq u+y\right)-P\left(T_{n} \leq u\right)\right| d y \leq C\left\{\gamma_{2 n}^{\rho}+1 / T\right\} .
$$

Therefore, combining (3.23) and (3.24), choosing $T=\gamma_{4 n}^{-1 / 4}$, and using the Esseen inequality (see [[18], Theorem 5.3]), we conclude that

$$
\begin{aligned}
& \sup _{u}\left|P\left(S_{n 111}^{\prime} \leq u\right)-P\left(T_{n} \leq u\right)\right| \\
\leq & \int_{-T}^{T}\left|\frac{\phi_{1}(t)-\psi_{1}(t)}{t}\right| d t+T \sup _{u} \int_{|\gamma| \leq c / T}\left|\tilde{G}_{n}(u+\gamma)-\tilde{G}_{n}(u)\right| d y \\
= & C\left\{\gamma_{2 n}^{\rho}+\gamma_{4 n}^{1 / 4}\right\} .
\end{aligned}
$$

\section{Some preliminary lemmas for $\hat{g}_{n}(t)$}

From the definition of $\hat{g}_{n}(t)$ in (1.5), We can decompose the sum into three parts:

$$
\begin{gathered}
S_{n g}:=\sigma_{n 2}^{-1}\left(\hat{g}_{n}(t)-\mathrm{E} \hat{g}_{n}(t)\right)=\sigma_{n 2}^{-1} \sum_{i=1}^{n} \varepsilon_{i} \int_{A_{i}} E_{m}(t, s) d s+\sigma_{n 2}^{-1} \sum_{i=1}^{n} x_{i}\left(\beta-\hat{\beta}_{n}\right) \int_{A_{i}} E_{m}(t, s) d s \\
-\sigma_{n 2}^{-1} \sum_{i=1}^{n} x_{i}\left(\beta-\mathrm{E} \hat{\beta}_{n}\right) \int_{A_{i}} E_{m}(t, s) d s=: H_{1 n}+H_{2 n}+H_{3 n} .
\end{gathered}
$$

Let us decompose the vector $H_{1 n}$ into two parts:

$$
H_{1 n}=\sigma_{n 2}^{-1} \sum_{i=1}^{n} \int_{A_{i}} E_{m}(t, s) d s\left(\sum_{j=-n}^{n} a_{j} e_{i-j}\right)+\sigma_{n 2}^{-1} \sum_{i=1}^{n} \int_{A_{i}} E_{m}(t, s) d s\left(\sum_{|j|>n} a_{j} e_{i-j}\right)=: H_{11 n}+H_{12 n}
$$

Where

$$
H_{11 n}=\sigma_{n 2}^{-1} \sum_{l=1-n}^{2 n}\left(\sum_{i=\max (1, l-n)}^{\min (n, n+l)} a_{i-l} \int_{A_{i}} E_{m}(t, s) d s\right) e_{l}=\sum_{l=1-n}^{2 n} M_{n l} .
$$

Similar to $S_{n 111}$ in (3.6), $H_{11 n}$ can be split as $H_{11 n}=H_{11 n}^{\prime}+H_{11 n}^{\prime \prime}+H_{11 n}^{\prime \prime \prime}$, where

$$
\begin{aligned}
& H_{11 n}^{\prime}=\sum_{w=1}^{k} y_{2 n w}, H_{11 n}^{\prime \prime}=\sum_{w=1}^{k} y_{2 n w^{\prime}}^{\prime} H_{11 n}^{\prime \prime \prime}=y_{2 n k+1}^{\prime} \\
& y_{2 n w}=\sum_{i=k_{w}}^{k_{w}+p-1} M_{n i}, y_{2 n w}^{\prime}=\sum_{i=l_{w}}^{l_{w}+q-1} M_{n i}, y_{2 n k+1}^{\prime}=\sum_{i=k(p+q)-n+1}^{2 n} M_{n i} .
\end{aligned}
$$


Then

$$
S_{n g}=H_{11 n}^{\prime}+H_{11 n}^{\prime \prime}+H_{11 n}^{\prime \prime \prime}+H_{2 n}+H_{3 n} .
$$

Set $T_{n 2}=\sum_{w=1}^{k} \eta_{2 n w}, B_{n 2}^{2}=\sum_{w=1}^{k} \operatorname{Var}\left(\eta_{2 n w}\right)$. Similarly to Lemmas 3.2-3.6, we have the following lemmas without proofs, except for Lemma 4.2.

Lemma 4.1. Suppose that the conditions in Theorem 2.2 are satisfied, then

$$
\begin{gathered}
\mathrm{E}\left(H_{11 n}^{\prime \prime}\right)^{2} \leq C \lambda_{1 n}, \mathrm{E}\left(H_{11 n}^{\prime \prime \prime}\right)^{2} \leq C \lambda_{2 n}, \mathrm{E} H_{12 n}^{2} \leq C \lambda_{3 n} ; \\
P\left(\left|H_{11 n}^{\prime \prime}\right| \geq \lambda_{1 n}^{1 / 3}\right) \leq C \lambda_{1 n}^{1 / 3}, P\left(\left|H_{11 n}^{\prime \prime \prime}\right| \geq \lambda_{2 n}^{1 / 3}\right) \leq C \lambda_{2 n}^{1 / 3}, P\left(\left|H_{12 n}\right| \geq \lambda_{3 n}^{1 / 3}\right) \leq C \lambda_{3 n}^{1 / 3} .
\end{gathered}
$$

Lemma 4.2. Let assumptions (A1)-(A7) be satisfied, then

$$
E\left|H_{2 n}\right|^{2+\delta} \leq c \lambda_{5 n}^{2+\delta}, P\left(\left|H_{2 n}\right|>\lambda_{5 n}^{(2+\delta) /(3+\delta)}\right) \leq \lambda_{5 n}^{(2+\delta) /(3+\delta)},\left|H_{3 n}\right| \leq c \lambda_{5 n} .
$$

Lemma 4.3. Under the conditions of Theorem 2.2, set $s_{n 2}^{2}=\sum_{w=1}^{k} \operatorname{Var}\left(\gamma_{2 n w}\right)$, then

$$
\left|s_{n 2}^{2}-1\right| \leq C\left(\lambda_{1 n}^{1 / 2}+\lambda_{2 n}^{1 / 2}+\lambda_{3 n}^{1 / 2}+u(q)\right)
$$

Lemma 4.4. Suppose that the conditions in Theorem 2.2 are satisfied, then

$$
\sup _{u}\left|P\left(T_{n 2} / B_{n 2} \leq u\right)-\Phi(u)\right| \leq c \lambda_{2 n}^{\rho} \text {. }
$$

Lemma 4.5. Suppose that the conditions in Theorem 2.2 are satisfied, then

$$
\sup _{u}\left|P\left(H_{11 n}^{\prime} \leq u\right)-P\left(T_{n 2} \leq u\right)\right| \leq C\left\{\lambda_{2 n}^{\rho}+\gamma_{4 n}^{1 / 4}\right\}
$$

Proof of Lemma 4.2. Similar to the proof of (A.8) in [6], we first verify that

$$
\lim _{n \rightarrow \infty} S_{n} / n=\lim _{n \rightarrow \infty} \frac{1}{n} \sum_{i=1}^{n} \tilde{x}_{i}^{2}=\sum, \quad \text { where } 0<\sum<\infty
$$

From (1.2), we write

$$
\begin{gathered}
\frac{1}{n} \sum_{i=1}^{n} \tilde{x}_{i}^{2}=\frac{1}{n} \sum_{i=1}^{n} u_{i}^{2}+\frac{1}{n} \sum_{i=1}^{n} \tilde{h}_{i}^{2}+\frac{1}{n} \sum_{i=1}^{n}\left(\sum_{j=1}^{n} u_{j} \int_{A_{j}} E_{m}\left(t_{i}, s\right) d s\right)^{2}+\frac{2}{n} \sum_{i=1}^{n} u_{i} \tilde{h}_{i} \\
-\frac{2}{n} \sum_{i=1}^{n} u_{i}\left(\sum_{j=1}^{n} u_{j} \int_{A_{j}} E_{m}\left(t_{i}, s\right) d s\right)-\frac{2}{n} \sum_{i=1}^{n} \tilde{h}_{i}\left(\sum_{j=1}^{n} u_{j} \int_{A_{j}} E_{m}\left(t_{i}, s\right) d s\right) \\
=L_{1 n}+L_{2 n}+L_{3 n}+2 L_{4 n}-2 L_{5 n}-2 L_{6 n} .
\end{gathered}
$$

By assumption (A2)(i) and Remark 2.1, we have

$$
L_{1 n} \rightarrow \sum, L_{2 n} \leq \max _{1 \leq i \leq n} \tilde{h}_{i}^{2}=O\left(n^{-1}+2^{-m}\right) \rightarrow 0,
$$


and by assumption (A2)(iii), Lemmas A.4 and A.5, we get that

$$
\begin{aligned}
L_{3 n} & \leq \frac{c}{n} \max _{1 \leq i, j \leq n} \int_{A_{j}}\left|E_{m}\left(t_{i}, s\right)\right| d s \max _{1 \leq j \leq n} \sum_{i=1}^{n}\left|\int_{A_{j}} E_{m}\left(t_{i}, s\right)\right| d s\left(\max _{1 \leq j \leq n}\left|\sum_{i=1}^{l} u_{j_{i}}\right|\right)^{2} \\
& =O\left(\frac{2^{m} \log ^{2} n}{n}\right) \rightarrow 0, \\
\left|L_{4 n}\right| & \leq \frac{c}{n} \max _{1 \leq i \leq n}\left|\tilde{h}_{i}\right| \cdot \max _{1 \leq l \leq n}\left|\sum_{i=1}^{l} u_{j_{i}}\right|=O\left(\frac{\log n}{2^{m} \sqrt{n}}\right) \rightarrow 0, \\
\left|L_{5 n}\right| & \leq \frac{c}{n} \max _{1 \leq i, j \leq n_{A_{j}}}\left|E_{m}\left(t_{i}, s\right)\right| d s \cdot \max _{1 \leq l \leq n}\left|\sum_{i^{\prime}=1}^{l} u_{j^{\prime}}\right| \cdot \max _{1 \leq l \leq n}\left|\sum_{i=1}^{l} u_{j_{i}}\right| \\
& =O\left(\frac{2^{m^{m}} \log ^{2} n}{n}\right) \rightarrow 0, \\
\left|L_{6 n}\right| & \leq \frac{c}{n} \max _{1 \leq i \leq n}\left|\tilde{h}_{i}\right| \cdot \max _{1 \leq j \leq n} \sum_{i=1}^{n} \int_{A_{j}}\left|E_{m}\left(t_{i}, s\right)\right| d s \cdot \max _{1 \leq l \leq n}\left|\sum_{i=1}^{l} u_{j_{i}}\right|=O\left(\frac{\log n}{2^{m} \sqrt{n}}\right) \rightarrow 0 .
\end{aligned}
$$

Therefore, from (4.4) to (4.6), we complete the proof of (4.3).

Recalling the fact that if $\xi_{n} \Rightarrow \xi \sim N(0,1)$ then $\mathrm{E}\left|\xi_{n}\right| \rightarrow \mathrm{E}|\xi|=\sqrt{2 / \pi}$ and $\mathrm{E}\left|\xi_{n}\right|^{2+\delta}$ $\rightarrow \mathrm{E}|\xi|^{2+\delta}<\infty$, by Theorem 2.1, Lemma 3.1 and relation (4.3), we deduce that

$$
\left|\beta-\mathrm{E} \hat{\beta}_{n}\right| \leq \mathrm{E}\left|\beta-\hat{\beta}_{n}\right| \leq O\left(\sigma_{n 1} / S_{n}^{2}\right)=O\left(n^{-1 / 2}\right),
$$

and

$$
\mathrm{E}\left|\hat{\beta}_{n}-\beta\right|^{2+\delta} \leq O\left(\left(\sigma_{n 1} / S_{n}^{2}\right)^{2+\delta}\right)=O\left(n^{-(1+\delta / 2)}\right) .
$$

Therefore, applying the Abel Inequality, by (A2)(iii) and (A4)(i), we get that

$$
\begin{aligned}
\left|H_{3 n}\right| & =\sigma_{n 2}^{-1}\left|\beta-\mathrm{E} \hat{\beta}_{n}\right| \cdot\left|\sum_{i=1}^{n} x_{i} \int E_{A_{i}} E_{m}(t, s) d s\right| \\
& \leq \sigma_{n 2}^{-1} n^{-1 / 2}\left(\sup _{0 \leq t \leq 1}|h(t)|+\max _{1 \leq i \leq n} \int_{A_{i}}\left|E_{m}(t, s)\right| d s \cdot \max _{1 \leq l \leq n}\left|\sum_{i=1}^{l} u_{j_{i}}\right|\right) \\
& \leq c\left(2^{-m / 2}+\sqrt{2^{m} / n} \log n\right)=c \lambda_{5 n},
\end{aligned}
$$

and

$$
\begin{aligned}
\mathrm{E}\left|H_{2 n}\right|^{2+\delta} & =\sigma_{n 2}^{-(2+\delta)} \mathrm{E}\left|\beta-\hat{\beta}_{n}\right|^{2+\delta} \cdot\left|\sum_{i=1}^{n} x_{i} \int_{A_{i}} E_{m}(t, s) d s\right|^{2+\delta} \\
& \leq c \sigma_{n 2}^{-(2+\delta)} n^{-(2+\delta) / 2}\left(\sup _{0 \leq t \leq 1}|\tilde{h}(t)|+\max _{1 \leq i \leq n} \int_{A_{i}}\left|E_{m}(t, s)\right| d s \cdot \max _{1 \leq l \leq n}\left|\sum_{i=1}^{l} u_{j_{i}}\right|\right)^{2+\delta} \\
& \leq c \lambda_{5 n}^{2+\delta} .
\end{aligned}
$$

By (4.8), it follows

$$
P\left(\left|H_{2 n}\right|>\lambda_{5 n}^{(2+\delta) /(3+\delta)}\right) \leq \lambda_{5 n}^{(2+\delta) /(3+\delta)} .
$$

Therefore, the proof is completed by (4.7)-(4.9). 


\section{Proofs of main results}

Proof of Theorem 2.1. Note that

$$
\begin{aligned}
\sup _{u}\left|P\left(S_{n 111}^{\prime} \leq u\right)-\Phi(u)\right| & \leq \sup _{u}\left|P\left(S_{n 111}^{\prime} \leq u\right)-P\left(T_{n} \leq u\right)\right|+\sup _{u}\left|P\left(T_{n} \leq u\right)-\Phi\left(u / s_{n}\right)\right| \\
& +\sup _{u}\left|\Phi\left(u / s_{n}\right)-\Phi(u)\right|=: J_{1 n}+J_{2 n}+J_{3 n} .
\end{aligned}
$$

By Lemmas 3.6, 3.5, and 3.4, we obtain, respectively

$$
\begin{aligned}
& J_{1 n} \leq C\left\{\gamma_{2 n}^{\rho}+\gamma_{4 n}^{1 / 4}\right\} \\
& J_{2 n}=\sup _{u}\left|P\left(T_{n} / s_{n} \leq u / s_{n}\right)-\Phi\left(u / s_{n}\right)=\sup _{u}\right| P\left(T_{n} / s_{n} \leq u\right)-\Phi(u) \mid \leq C \gamma_{4 n}^{\rho},
\end{aligned}
$$

and

$$
J_{3 n} \leq C\left|s_{n}^{2}-1\right| \leq C\left(\gamma_{1 n}^{1 / 2}+\gamma_{2 n}^{1 / 2}+\gamma_{3 n}^{1 / 2}+u(q)\right)
$$

Hence, from (5.1) to (5.4), we get that

$$
\sup _{u}\left|P\left(S_{n 111}^{\prime} \leq u\right)-\Phi(u)\right| \leq C\left\{\sum_{i=1}^{3} \gamma_{i n}^{1 / 2}+u(q)+\gamma_{2 n}^{\rho}+\gamma_{4 n}^{1 / 4}\right\} .
$$

Therefore, according to Lemma A.3, relations (3.8), (3.12), and (5.5), we obtain

$$
\begin{aligned}
& \sup _{u}\left|P\left(S_{n \beta} \leq u\right)-\Phi(u)\right| \\
&=\sup _{u}\left|P\left(S_{n 111}^{\prime}+S_{n 111}^{\prime \prime}+S_{n 111}^{\prime \prime \prime}+S_{n 112}+S_{n 12}+S_{n 13}+S_{n 2}+S_{n 3} \leq u\right)-\Phi(u)\right| \\
& \leq C\left\{\sup _{u}\left|P\left(S_{n 111}^{\prime} \leq u\right)-\Phi(u)\right|\right. \\
&\left.+\sum_{i=1}^{3} \gamma_{i n}^{1 / 3}+P\left(\left|S_{n 111}^{\prime \prime}\right| \geq \gamma_{1 n}^{1 / 3}\right)+P\left(\left|S_{n 111}^{\prime \prime \prime}\right| \geq \gamma_{2 n}^{1 / 3}\right)+P\left(\left|S_{n 112}\right| \geq \gamma_{3 n}^{1 / 3}\right)\right\} \\
&\left.+\left(n^{-1}+2^{-m}\right)^{2 / 3}+\left(2^{m} n^{-1} \log ^{2} n\right)^{1 / 3}\right)+2^{-m} \log n+n^{1 / 2} 2^{-2 m} \\
&+P\left(\left|S_{n 12}\right| \geq\left(n^{-1}+2^{-m}\right)^{2 / 3}\right)+P\left(\left|S_{n 13}\right| \geq\left(2^{m} n^{-1} \log ^{2} n\right)^{1 / 3}\right) \\
&+P\left(\left|S_{n 21}\right| \geq\left(2^{m} n^{-1} \log ^{2} n\right)^{1 / 3}\right)+P\left(\left|S_{n 22}\right| \geq\left(n^{-1}+2^{-m}\right)^{2 / 3}\right) \\
&+P\left(\left|S_{n 23}\right| \geq\left(2^{m} n^{-1} \log ^{2} n\right)^{1 / 3}\right) \\
& \leq c\left(\gamma_{1 n}^{1 / 2}+\gamma_{2 n}^{1 / 2}+\gamma_{3 n}^{1 / 2}+u(q)+\gamma_{2 n}^{\rho}+\gamma_{4 n}^{1 / 4}+\sum_{i=1}^{3} \gamma_{i n}^{1 / 3}\right) \\
&+c\left(2^{-\frac{2 m}{3}}+\left(2^{m} n^{-1} \log ^{2} n\right)^{1 / 3}+2^{-m} \log n+n^{1 / 2} 2^{-2 m}\right) \\
& \leq c\left(\sum_{i=1}^{3} \gamma_{i n}^{1 / 3}+u(q)+\gamma_{2 n}^{\rho}+\gamma_{4 n}^{1 / 4}\right)+c\left(2^{-2 m / 3}+\left(2^{m} n^{-1} \log ^{2} n\right)^{1 / 3}+2^{-m} \log n+n^{1 / 2} 2^{-2 m}\right) \\
&= O\left(\mu_{n}(\rho, p)\right)+O\left(v_{n}(m)\right) .
\end{aligned}
$$

This completes the proof of the theorem.

Proof of Corollary 2.1. Let $p=\left[n^{\tau}\right], q=\left[n^{2 \tau-1}\right]$, where $\tau=\frac{3 \lambda+7}{6 \lambda+7}$, then

$$
\begin{aligned}
& \gamma_{1 n}^{1 / 3}=\gamma_{2 n}^{1 / 3}=O\left(n^{(\tau-1) / 3}\right)=O\left(n^{-\frac{\lambda}{6 \lambda+7}}\right), \gamma_{4 n}^{1 / 4}=O\left(n^{-\frac{\lambda}{6 \lambda+7}}\right), \\
& \lambda_{3 n}^{1 / 3}=n^{-1 / 4}(\log n)^{3 / 4}\left(\sup _{n \geq 1} n^{7 / 8}(\log n)^{-9 / 8} \sum_{|j|>n}\left|a_{j}\right|\right)^{2 / 3}=O\left(n^{-1 / 4}(\log n)^{3 / 4}\right) .
\end{aligned}
$$


By $\delta>1 / 3, \lambda \geq \max \left\{\frac{2+\delta}{\delta}, \frac{7 \delta+14}{6 \delta-2}\right\}$, we get

$$
u(q)=O\left(q^{-\lambda \delta /(2+\delta)+1}\right)=O\left(n^{-\frac{7}{6 \lambda+7}\left(\frac{\lambda \delta}{2+\delta}-1\right)}\right) \leq O\left(n^{-\frac{\lambda}{6 \lambda+7}}\right)
$$

Thus, we have that

$$
\mu_{n}(\rho, p)=O\left(n^{-\frac{\lambda}{6 \lambda+7}}\right), v_{n}(m)=O\left(n^{-\frac{1}{5}}\right)
$$

Therefore, (2.2) follows from Theorem 2.1 directly.

Proof of Theorem 2.2. Analogous to the Proof of Theorem 2.1, we write

$$
\begin{aligned}
& \sup _{u}\left|P\left(H_{11 n}^{\prime} \leq u\right)-\Phi(u)\right| \\
& \leq \sup _{u}\left|P\left(H_{11 n}^{\prime} \leq u\right)-P\left(T_{n 2} \leq u\right)\right|+\sup _{u}\left|P\left(T_{n 2} \leq u\right)-\Phi\left(u / s_{n 2}\right)\right| \\
& +\sup _{u}\left|\Phi\left(u / s_{n 2}\right)-\Phi(u)\right|=: J_{1 n}^{\prime}+J_{2 n}^{\prime}+J_{3 n}^{\prime} .
\end{aligned}
$$

By Lemmas 4.5, 4.4, and 4.3, we obtain, respectively

$$
\begin{aligned}
& J_{1 n}^{\prime} \leq C\left\{\lambda_{2 n}^{\rho}+\gamma_{4 n}^{1 / 4}\right\}, J_{2 n}^{\prime}=\sup _{u}\left|P\left(T_{n 2} / s_{n 2} \leq u\right)-\Phi(u)\right| \leq C \lambda_{2 n^{\prime}}^{\rho} \\
& J_{3 n}^{\prime} \leq C\left|s_{n 2}^{2}-1\right| \leq C\left(\lambda_{1 n}^{1 / 2}+\lambda_{2 n}^{1 / 2}+\lambda_{3 n}^{1 / 2}+u(q)\right) .
\end{aligned}
$$

Therefore, from (5.6) to (5.8), we have

$$
\sup _{n}\left|P\left(H_{11 n}^{\prime} \leq u\right)-\Phi(u)\right| \leq C\left\{\sum_{i=1}^{3} \lambda_{i n}^{1 / 2}+u(q)+\lambda_{2 n}^{\rho}+\lambda_{4 n}^{1 / 4}\right\}
$$

Thus, according to Lemmas A.3, 4.1, and 4.2, we obtain

$$
\begin{aligned}
& \sup _{u}\left|P\left(S_{n g} \leq u\right)-\Phi(u)\right| \\
= & \sup _{u}\left|P\left(H_{11 n}^{\prime}+H_{11 n}^{\prime \prime}+H_{11 n}^{\prime \prime \prime}+H_{12 n}+H_{n 2}+H_{n 3} \leq u\right)-\Phi(u)\right| \\
\leq & C \sup _{u}\left|P\left(H_{11 n}^{\prime} \leq u\right)-\Phi(u)\right|+\sum_{i=1}^{3} \lambda_{i n}^{1 / 3}+P\left(\left|H_{11 n}^{\prime \prime}\right| \geq \lambda_{1 n}^{1 / 3}\right)+P\left(\left|H_{11 n}^{\prime \prime \prime}\right| \geq \lambda_{2 n}^{1 / 3}\right) \\
& \left.+P\left(\left|H_{12 n}\right| \geq \lambda_{3 n}^{1 / 3}\right)+\lambda_{5 n}+\lambda_{5 n}^{(2+\delta) /(3+\delta)}+P\left(\left|H_{12 n}\right|>\lambda_{5 n}^{(2+\delta) /(3+\delta)}\right)\right\} \\
\leq & c\left(\sum_{i=1}^{3} \lambda_{i n}^{1 / 2}+u(q)+\lambda_{2 n}^{\rho}+\lambda_{4 n}^{1 / 4}+\sum_{i=1}^{3} \lambda_{i n}^{1 / 3}+\lambda_{5 n}^{(2+\delta) /(3+\delta)}\right) \\
\leq & c\left(\sum_{i=1}^{3} \lambda_{i n}^{1 / 3}+u(q)+\lambda_{2 n}^{\rho}+\lambda_{4 n}^{1 / 4}+\lambda_{5 n}^{(2+\delta) /(3+\delta)}\right) .
\end{aligned}
$$

Now we complete the Proof of Theorem 2.2.

Proof of Corollary 2.2. Let $p=\left[n^{\tau}\right], q=\left[n^{2 \tau-1}\right]$, where $\tau=\frac{1}{2}+\frac{8 \theta-1}{2(6 \lambda+7)}$. We can get $\tau<\theta$ by $\frac{\lambda+1}{2 \lambda+1}<\theta$. By this, we can obtain 


$$
\begin{aligned}
& \lambda_{1 n}^{1 / 3}=\lambda_{2 n}^{1 / 3}=O\left(n^{-(\theta-\tau) / 3}\right)=O\left(n-\left(\frac{\lambda(2 \theta-1)+(\theta-1)}{6 \lambda+7}\right)\right), \lambda_{4 n}^{1 / 4}=O\left(n-\left(\frac{\lambda(2 \theta-1)+(\theta-1)}{6 \lambda+7}\right)\right), \\
& \lambda_{3 n}^{1 / 3}=n^{-1 / 4}(\log n)^{3 / 4}\left(\sup _{n \geq 1} n^{7 / 8}(\log n)^{-9 / 8} \sum_{|j|>n}\left|a_{j}\right|\right)^{2 / 3}=O\left(n^{-1 / 4}(\log n)^{3 / 4}\right) .
\end{aligned}
$$

And by $\delta>2 / 3$, and $\theta \leq 3 / 4$, we have

$$
\lambda_{5 n}^{(2+\delta) /(3+\delta)}=O\left(n^{-\frac{\theta}{2} \cdot \frac{2+\delta}{3+\delta}}\right) \leq O\left(n^{-\frac{4}{11} \theta}\right) \leq O\left(n^{-\frac{4 \lambda+4}{22 \lambda+11}}\right) .
$$

Since $\lambda>\frac{(2+\delta)(9 \theta-2)}{2 \theta(3 \delta-2)+2}$, we can obtain

$$
\frac{(8 \theta-1)(\lambda \delta-\delta-2)}{(7+6 \lambda)(2+\delta)}>\frac{\lambda(2 \theta-1)+(\theta-1)}{6 \lambda+7} .
$$

By this we have

$$
u(q)=O\left(q^{-\lambda \delta /(2+\delta)+1}\right)=O\left(n^{-\frac{(8 \theta-1)(\lambda \delta-\delta-2)}{(7+6 \lambda)(2+\delta)}}\right)=O\left(n^{-\frac{\lambda(2 \theta-1)+(\theta-1)}{6 \lambda+7}}\right) .
$$

Therefore, (2.3) follows from Theorem 2.2 directly.

\section{Appendix}

Lemma A.1. [8,9] Let $\left\{b_{j}: j \geq 1\right\}$ be a real sequence.

(i) Let $\left\{X_{j}: j \geq 1\right\}$ be an $\alpha$-mixing sequence of random variables with $E\left|X_{i}\right|^{2+\delta}<\infty, \delta>0$, then $E\left(\sum_{j=1}^{n} b_{j} X_{j}\right)^{2} \leq\left(1+20 \sum_{m=1}^{n} \alpha^{\delta /(2+\delta)}(m)\right) \sum_{j=1}^{n} b_{j}^{2}\left\|X_{j}\right\|_{2+\delta}^{2}, \forall n \geq$

(ii) Let $r>2, \delta>0$, and $\left\{X_{i}, i \geq 1\right\}$ be an $\alpha$-mixing sequence of random variables with $E X_{i}=0$ and $E\left|X_{i}\right|^{r+\delta}<\infty$. Suppose that $\theta>r(r+\delta) /(2 \delta)$ and $\alpha(n) \leq C n^{-\theta}$ for some $C$ $>0$. Then, for any $\varepsilon>0$, there exists a positive constants $K=K(\varepsilon, r, \delta, \theta, C)<\infty$ such that

$$
E \max _{1 \leq j \leq n}\left|\sum_{i=1}^{j} X_{i}\right|^{r} \leq K\left\{n^{\varepsilon} \sum_{i=1}^{n} E\left|X_{i}\right|^{r}+\left(\sum_{i=1}^{n}\left\|X_{i}\right\|_{r+\delta}^{2}\right)^{r / 2}\right\} .
$$

Lemma A.2. [10] Let $\left\{X_{j}: j \geq 1\right\}$ be an $\alpha$-mixing sequence of random variables, $p$ and, $q$ are two positive integers. Let $\eta_{l}:=\sum_{j=(l-1)(p+q)+1}^{(l-1)(p+q)+p} X_{j}$ for $1 \leq l \leq k$. If $r>0, s>$ 0 and $\frac{1}{r}+\frac{1}{s}=1$, then

$$
\left|E \exp \left(i t \sum_{l=1}^{k} \eta_{l}\right)-\prod_{l=1}^{k} E \exp \left(i t \eta_{l}\right)\right| \leq C|t| \alpha^{1 / s}(q) \sum_{l=1}^{k}\left\|\eta_{l}\right\|_{r} .
$$

Lemma A.3. [14] Suppose that $\left\{\zeta_{n}: n \geq 1\right\},\left\{\eta_{n}: n \geq 1\right\}$ and $\left\{\xi_{n}: n \geq 1\right\}$ are random sequences, $\left\{\gamma_{n}: n \geq 1\right\}$ is a positive constant sequence with $\gamma_{n} \rightarrow 0$. If $\sup _{u}\left|F_{\zeta_{n}}(u)-\Phi(u)\right| \leq C \gamma_{n}$, then for any $\varepsilon_{1}>0$, and $\varepsilon_{2}>0$ 


$$
\sup _{u}\left|F_{\zeta_{n}+\eta_{n}+\xi_{n}}(u)-\Phi(u)\right| \leq C\left\{\gamma_{n}+\varepsilon_{1}+\varepsilon_{2}+P\left(\left|\eta_{n}\right| \geq \varepsilon_{1}\right)+P\left(\left|\xi_{n}\right| \geq \varepsilon_{2}\right)\right\}
$$

Lemma A.4. [19] Under assumptions (A5)-(A6), we have

(i) $\left|\int_{A_{i}} E_{m}(t, s) d s\right|=O\left(\frac{2^{m}}{n}\right), \quad i=1,2, \ldots, n$; (ii) $\sum_{i=1}^{n}\left(\int_{A_{i}} E_{m}(t, s) d s\right)^{2}=O\left(\frac{2^{m}}{n}\right)$;

(iii) $\sup _{m} \int_{0}^{1}\left|E_{m}(t, s) d s\right| \leq C$; (iv) $\sum_{i=1}^{n}\left|\int_{A_{i}} E_{m}(t, s) d s\right| \leq C$.

Lemma A.5. [20] Under assumptions (A5) and (A7), we have

(i) $\max _{1 \leq i \leq n} \sum_{j=1}^{n} \int_{A_{j}}\left|E_{m}\left(t_{i}, s\right)\right| d s \leq c$; (ii) $\max _{1 \leq i \leq n} \sum_{j=1}^{n} \int_{A_{i}}\left|E_{m}\left(t_{j}, s\right)\right| d s \leq c$.

\section{Acknowledgements}

The authors are grateful to the two anonymous referees for providing detailed list of comments and suggestions which greatly improved an earlier version of this article. This work is partially supported by the National Science Foundation of China (No. 11061029) and the Natural Science Foundation of Jiangxi Province (No. 2008GZS0046).

\section{Author details}

'School of Mathematics and Statistics, Wuhan University, Wuhan 430072, China ${ }^{2}$ College of Mathematical Sciences, Guangxi Teachers Education University, Nanning 530023, China ${ }^{3}$ Department of Mathematics, Shangrao Normal University, Shangrao 334001, China

\section{Authors' contributions}

The two authors contributed equally to this work. All authors read and approved the final manuscript.

\section{Competing interests}

The authors declare that they have no competing interests.

Received: 25 September 2011 Accepted: 27 February 2012 Published: 27 February 2012

\section{References}

1. Engle, R, Granger, C, Rice, J, Weiss, A: Nonparametric estimates of the relation between weather and electricity sales. J Am Stat Assoc. 81, 310-320 (1986). doi:10.2307/2289218

2. Chen, H, Shiah, J: Data-driven efficient estimation for a partially linear model. Ann Stat. 22, 211-237 (1994). doi:10.1214/ aos/1176325366

3. Donald, G, Dewey, K: Series estimation of semi linear models. J Multivar Anal. 50, 30-40 (1994). doi:10.1006/ jmva.1994.1032

4. Hamilton, SA, Truong, YK: Local linear estimation in partly linear models. J Multivar Anal. 60, 1-19 (1997). doi:10.1006/ jmva.1996.1642

5. Sun, $\mathrm{XQ}, \mathrm{You}, \mathrm{JH}$, Chen, GM, Zhou, $\mathrm{X}$ : Convergence rates of estimators in partial linear regression models with $\mathrm{MA}(\infty)$ error process. Commun Stat Theory Methods. 31, 2251-2273 (2002). doi:10.1081/STA-120017224

6. Liang, HY, Fan, GL: Berry-Esseen type bounds of estimators in a semiparametric model with linear process errors. J Multivar Anal. 100(1):1-15 (2009). doi:10.1016/j.jmva.2008.03.006

7. Lin, ZY, Lu, CR: Limit Theory for Mixing Dependent Random Variables. Science Press, Beijing (1996)

8. Yang, SC: Moment bounds for strong mixing sequences and their application. J Math Res Expos. 20(3):349-359 (2000)

9. Yang, SC: Maximal moment inequality for partial sums of strong mixing sequences and application. Acta Math Sin. 26B(3):1013-1024 (2007)

10. Yang, SC, Li, YM: Uniformly asymptotic normality of the regression weighted estimator for strong mixing samples. Acta Math $\operatorname{Sin} .49 \mathrm{~A}(5): 1163-1170(2006)$

11. Xing, GD, Yang, SC: On the Maximal inequalities for partial sums of strong mixing random variables with applications. Thai J Math. 9(1):11-19 (2011)

12. Xing, GD, Yang, SC, Chen, A: A maximal moment inequality for a-mixing sequences and its applications. Stat Probab Lett. 79, 1429-1437 (2009). doi:10.1016/j.spl.2009.02.016

13. Xing, GD, Yang, SC, Liu, Y, Yu, KM: A note on the Bahadur representation of sample quantiles for a-mixing random variables. Monatsh Math (2011). doi:10.1007/s00605-011-0334-0

14. Li, YM, Wei, CD, Xing, GD: Berry-Esseen bounds for wavelet estimator in a regression model with linear process errors. Stat Probab Lett. 81(1):103-110 (2011). doi:10.1016/j.spl.2010.09.024

15. Antoniadis, A, Gregoire, G, McKeague, IW: Wavelet methods for curve estimation. J Am Stat Assess. 89, 1340-1352 (1994). doi:10.2307/2290996

16. Negiahi, $\mathrm{H}$ : The rate of convergence to normality for strong mixing sequences of random variables. Sci Rep Yokohama Natl Univ Sect 24(11):17-25 (1977). 1 Math. Phys. Chem

17. You, JH, Chen, $\mathrm{M}, \mathrm{Chen}, \mathrm{G}$ : Asymptotic normality of some estimators in a fixed-design semiparametric regression model with linear time series errors. J Syst Sci Complex. 17(4):511-522 (2004)

18. Petrov, W: Limit Theory for Probability Theory. Oxford University Press, New York (1995) 
19. Li, YM, Guo, JH: Asymptotic normality of wavelet estimator for strong mixing errors. J Korean Stat Soc. 38, 383-390 (2009). doi:10.1016/j.jkss.2009.03.002

20. Xue, LG: Rates of random weighting approximation of wavelet estimates in semipara-metric regression model. Acta Math Appl Sin. 26, 11-25 (2003)

doi:10.1186/1029-242X-2012-44

Cite this article as: Wei and Li: Berry-Esseen bounds for wavelet estimator in semiparametric regression model with linear process errors. Journal of Inequalities and Applications 2012 2012:44.

Submit your manuscript to a SpringerOpen ${ }^{\odot}$ journal and benefit from:

- Convenient online submission

- Rigorous peer review

- Immediate publication on acceptance

- Open access: articles freely available online

- High visibility within the field

- Retaining the copyright to your article

Submit your next manuscript at $\gg$ springeropen.com 